\title{
Plasma Fibrinogen Acts as a Predictive Factor for Pathologic Complete Response to Neoadjuvant Chemotherapy in Breast Cancer: A Retrospective Study of 1035 Chinese Breast Cancer Patients
}

\section{Yihua Wang}

Chongqing Medical University First Affiliated Hospital

\section{Yu Wang}

Chongqing Medical University First Affiliated Hospital

Rui Chen

Chongqing Medical University First Affiliated Hospital

\section{Zhenrong Tang}

Chongqing Medical University First Affiliated Hospital

\section{Yang Peng}

Chongqing Medical University First Affiliated Hospital

Shengchun Liu ( $\square$ liushengchun1968@163.com)

Chongqing Medical University First Affiliated Hospital https://orcid.org/0000-0002-9933-3643

\section{Research article}

Keywords: breast cancer, coagulation factor, fibrinogen, pathologic complete response, predictive ability

Posted Date: September 2nd, 2020

DOI: https://doi.org/10.21203/rs.3.rs-66857/v1

License: (c) (i) This work is licensed under a Creative Commons Attribution 4.0 International License.

Read Full License 


\section{Abstract}

Background: The aim of this study was to evaluate the relationship between pre-treatment plasma fibrinogen (Fib) level and pathological complete response (pCR) to neoadjuvant chemotherapy (NAC) in breast cancer patients and to assess the role of plasma Fib as a predictive factor.

Methods: Data from 1035 consecutive patients with invasive breast cancer who received NAC and subsequent surgery were retrospectively analysed. Both univariate and multivariate analyses were performed to identify clinicopathological factors associated with pCR to NAC.

Results: The median value of Fib, rather than other plasma coagulation parameters, was significantly increased in non-pCR patients compared with pCR patients $(P=0.008)$. Based on the cut-off value estimated by the ROC curve analysis, samples were divided into low or high Fib groups (Fib $<3.145 \mathrm{~g} / \mathrm{L}$ or $\geq 3.145 \mathrm{~g} / \mathrm{L}$ ). Low Fib status was significantly associated with premenopausal or perimenopausal status $(P<0.001), \leq 5 \mathrm{~cm}$ tumour size $(P=0.001)$, positive hormone receptor status $(P=0.003)$ and $>14 \%$ Ki67 index $(P=0.028)$. Adjusted for other clinicopathologic factors in the multivariate logistic regression model, low Fib status was strongly associated with pCR to NAC $(\mathrm{OR}=2.365,95 \% \mathrm{Cl}=1.354-4.133, \mathrm{P}=$ $0.002)$.

Conclusions: This study demonstrates that low pre-treatment plasma Fib (Fib $<3.145 \mathrm{~g} / \mathrm{L}$ ) is an independent predictive factor for pCR to NAC in breast cancer patients.

\section{Background}

Breast cancer is currently the most common cancer ( $25 \%$ of all cancer cases) and is one of the leading causes of cancer-related death (15\% of cancer deaths) among females worldwide [1]. Neoadjuvant chemotherapy (NAC) has become an integral part of the systematic treatment of breast cancer; NAC is used to convert unresectable to resectable cancers, to increase the rate of success for breast-conserving surgery and to evaluate the response to chemotherapy regimens before surgery [2]. It has been recognized that patients with a pathological complete response (pCR) after NAC have a significant survival advantage over those with residual invasive disease [3, 4].

Novel predictive biomarkers that can predict the pCR prior to NAC are valuable for making individualized treatment decisions and for maximizing efficacy in breast cancer patients. A series of studies have suggested an association between the haemostatic system and tumour biology [5, 6]. Different molecular mechanisms can cause the onset of a hypercoagulable state, and hypercoagulability in cancer patients has been implicated in angiogenesis, tumour cell invasion, tumour progression, and metastatic spread [6]. Subclinical hypercoagulable states have also been demonstrated in breast cancer patients [7, 8]. Fibrinogen (Fib), a key coagulation factor mainly synthesized by the liver, could be converted to fibrin by activated thrombin[9]. Previous studies have demonstrated that increased plasma Fib levels are frequently observed in cancer patients, and Fib has been shown to play a vital role in tumorigenesis and to contribute to angiogenesis, stroma formation, and hematogenous metastasis of tumour cells $[10,11]$. 
Recent studies have shown that elevated pre-treatment plasma Fib levels are associated with poor prognosis in breast cancer [12-14]. However, there have been very few studies on the correlation between the pre-treatment plasma Fib level and the PCR to NAC in breast cancer patients. The aim of the present study was to evaluate the relationship between the pre-treatment plasma Fib level and the pCR to NAC in breast cancer patients and assess the role of Fib as a predictive factor.

\section{Methods}

\section{Patients and treatments}

This retrospective study includes 1312 invasive breast cancer patients whose diagnoses were confirmed by histology and who received NAC from April 2012 to March 2019. The exclusion criteria for all participants were as follows: (1) patients with distant metastasis, bilateral breast cancer or male breast cancer, (2) patients with concurrent liver diseases, autoimmune diseases, haematological diseases or continuous anticoagulant treatment, (3) patients who were pregnant or had previous cancer or concomitant cancer, (4) patients who had radiotherapy, chemotherapy or surgery in the previous 3 months, (5) patients who received 22 cycles of NAC or no surgery, or (6) patients with incomplete data. Ultimately, 1035 patients were eligible for analysis (Figure 1). Medical records were reviewed to collect pre-treatment clinical data, such as age, menopausal status, tumour size, lymph node involvement, clinical stage (the 7th edition of the American Joint Committee on Cancer TNM Staging System) [15], histological subtype, oestrogen receptor (ER) status, progesterone receptor (PR) status, human epidermal growth factor receptor 2 (HER2) status, Ki67 index, and cycles of NAC. All patients received at least 2 but not more than 8 cycles of preoperative treatment with the TEC regimen: cyclophosphamide $\left(500 \mathrm{mg} / \mathrm{m}^{2}\right)$, epirubicin $\left(75 \mathrm{mg} / \mathrm{m}^{2}\right)$, and docetaxel $\left(75 \mathrm{mg} / \mathrm{m}^{2}\right)$ every 3 weeks. In our study, trastuzumab treatment was not used in patients with HER2-positive status in neoadjuvant set, due to the limitations of the medical security system.

\section{Ethical statements}

This research was conducted ethically in accordance with the World Medical Association Declaration of Helsinki and was approved by the Ethics Committee of our hospital (No. 2020-59), who deemed that written informed consent was not necessary due to the retrospective nature of the research.

\section{Blood sample analysis}

Samples of $5 \mathrm{~mL}$ venous blood were collected in tubes with sodium citrate before NAC for the plasma coagulation test. The obtained blood samples were processed within $24 \mathrm{~h}$ to detect the plasma coagulation parameters, including Fib, prothrombin time (PT), prothrombin time ratio (PTR), international normalized ratio (INR), activated partial thromboplastin time (APTT), prothrombin activity (PTA), thrombin 
time (TT), fibrinogen degradation product (FDP), and D-dimer (DD) with a Sysmex CS5100 automatic coagulation analyser (Sysmex Corporation, Kobe, Japan) in centre laboratory of our hospital.

\section{Immunohistochemical staining and scoring}

All breast cancer specimens were confirmed by core needle biopsy and tested using immunohistochemistry to determine the tumour subtype. According to the 2011 St. Gallen consensus,[16] the ER and PR status were considered positive if $>1 \%$ tumour cells were stained and the HER2 status was considered positive if $>10 \%$ of the tumour cells exhibited a $3+$ score by $\mathrm{IHC}$ or a $>2.2$-fold change compared to the expression of CEP17 in tumour cells via fluorescence in situ hybridization. Regarding Ki67, between 400 and 500 cells were counted to calculate the percentage of positive tumour cell nuclei, including hot spots, and $14 \%$ was defined as the optimal cut-off value. Hormone receptor (HR) positive was defined as ER and/or PR positive, while HR negative is defined as ER and PR negative. Based on HR status and HER2 status, the patients were classified according to the following subtypes: HR (+) HER2 (-), HR (+) HER2 (+), HR (-) HER2 (+), and HR (-) HER2 (-).

\section{Evaluation of chemotherapy response}

A pCR was defined as the absence of residual invasive tumour lesions in any breast tissue and lymph node (ypTOypNO or ypTO/is ypNO) [17]. Participants were classified into the pCR group and the non-pCR group.

\section{Statistical methods}

Data were analysed using SPSS (version 25.0) software (SPSS Inc., Chicago, IL, USA). The levels of plasma coagulation parameters were expressed as the median and interquartile range (IQR) because they are nonnormally distributed continuous variables, and the levels in the PCR group and the non-pCR group were compared by the Mann-Whitney $U$ test and Wilcoxon signed-rank test. Receiver operating characteristic (ROC) curve analysis and the Youden index were used to calculate the optimal cut-off value for pre-treatment plasma Fib levels. Categorical variables were represented as numbers and percentages and compared via Chi-square and Fisher's tests. Significant factors for pCR in univariate analyses were included in the multivariate logistic regression model with a forward LR method. Odds ratios (ORs) and $95 \%$ confidence intervals (Cls) with two-sided $\mathrm{P}$ values were used. Statistical significance was defined as a two-sided $\mathrm{P}$ value $<0.05$.

\section{Results}

\section{Patient characteristics}


A total of 1312 invasive breast cancer patients whose diagnoses were confirmed by histology and who received NAC before surgery in our institution were enrolled, and 1035 patients were eligible for analysis (Figure 1). The baseline patient characteristics were shown in Table 1. The median age of all the patients was 49 years (IQR, 43-56 years). In total, 348 patients (33.6\%) were premenopausal, 401 patients (38.7\%) were perimenopausal, and 286 patients (27.6\%) were postmenopausal. The most common

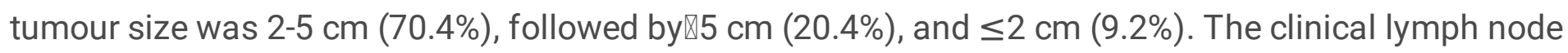
involvement status was described as follows: N0 (36.1\%), N1 (47.1\%), and N2-3 (16.8\%). The most of patients $(69.8 \%)$ were categorized as being in stage II, according to the TNM staging system. Most patients (91.4\%) received 4 chemotherapy cycles, and ductal (96.7\%) was the most frequent histological subtype. The subtype distribution was as follows: $35.9 \%$ for HR (+) HER2 (-), 21.0\% for HR (+) HER2 (+), $21.5 \%$ for HR (-) HER2 (+), $15.5 \%$ for HR (-) HER2 (-), and $6.1 \%$ for unknown. A total of 128 patients $(12.4 \%)$ achieved pCR to NAC. 
Table 1

Baseline characteristics ( $n=1035)$.

\section{Characteristics}

Age (y)

$\leq 50$

$>50$

Menstrual status

Premenopausal

Perimenopausal

Postmenopausal

Tumour size (cm)

$\leq 2$

2-5

$>5$

Lymph node involvement

NO

N1

N2-3

\section{Clinical stage}

।

II

III

Histologic subtype

Ductal

Lobular

Other

Unknown

ER status

Positive

Negative
Number of cases

585 (56.5\%)

450 (43.5\%)

$348(33.6 \%)$

$401(38.7 \%)$

$286(27.6 \%)$

95 (9.2\%)

$729(70.4 \%)$

$211(20.4 \%)$

$374(36.1 \%)$

$487(47.1 \%)$

$174(16.8 \%)$

39 (3.8\%)

$722(69.8 \%)$

274 (26.5\%)

1001 (96.7\%)

$14(1.4 \%)$

$16(1.5 \%)$

$4(0.4 \%)$

$620(59.9 \%)$

$415(40.1 \%)$ 


\section{PR status}

Positive

Negative

\section{HER2 status}

Negative

Positive

Unknown

Ki67 status (\%)

$\leq 14$

14-30

$>30$

\section{Subtype}

HR (+) HER2 (-)

HR (+) HER2 (+)

HR (-) HER2 (+)

HR (-) HER2 (-)

Unknown

Chemotherapy cycles

$<4$

4

$>4$

\section{Responder}

PCR

Non-pCR
455 (44.0\%)

580 (56.0\%)

440 (42.5\%)

532 (51.4\%)

$63(6.1 \%)$

277 (26.8\%)

$477(46.1 \%)$

$281(27.1 \%)$

372 (35.9\%)

217 (21.0\%)

$223(21.5 \%)$

160 (15.5\%)

$63(6.1 \%)$

18 (1.7)

946 (91.4)

$71(6.9)$

Abbreviations: ER, oestrogen receptor; PR, progesterone receptor; HER2, human epidermal growth factor receptor 2; HR, hormone receptor; $\mathrm{PCR}$, pathological complete response.

\section{Association between coagulation factors and pCR}

The plasma coagulation parameters were compared between the pCR group and the non-pCR group (Table 2). The median value of Fib was significantly increased in non-pCR patients compared with $\mathrm{pCR}$ 
patients (3.05 (2.63-3.48) g/L vs 2.90 (2.53-3.28) g/L, P = 0.008) (Figure 2A). However, no significant differences in PT, PTR, INR, APTT, PTA, TT, FDP, and DD were noted between the pCR group and the nonpCR group (all $P>0.05$ ).

Table 2

Coagulation parameters comparison between non-pCR group and $\mathrm{pCR}$ group.

\begin{tabular}{|c|c|c|c|c|}
\hline \multirow[b]{2}{*}{ Factors } & \multicolumn{4}{|l|}{ Median (IQR) } \\
\hline & Total $(n=1035)$ & $\operatorname{pCR}(n=128)$ & Non-pCR (n=907) & $P$ value \\
\hline PT & $12.80(12.40-13.30)$ & $12.80(12.40-13.20)$ & $12.80(12.40-13.30)$ & 0.933 \\
\hline PTR & $0.97(0.94-1.01)$ & $0.97(0.94-1.01)$ & $0.97(0.94-1.01)$ & 0.865 \\
\hline INR & $0.96(0.92-1.01)$ & $0.96(0.92-1.01)$ & $0.96(0.92-1.01)$ & 0.758 \\
\hline APTT & $35.50(33.20-37.80)$ & $35.50(33.40-37.88)$ & $35.50(33.20-37.80)$ & 0.795 \\
\hline PTA & $107.00(99.00-116.00)$ & $108.00(99.00-115.00)$ & $107.00(98.00-116.00)$ & 0.818 \\
\hline TT & $16.60(16.00-17.20)$ & $16.80(16.30-17.30)$ & $16.60(16.00-17.20)$ & 0.052 \\
\hline Fib & $3.04(2.63-3.45)$ & $2.90(2.53-3.28)$ & $3.05(2.63-3.48)$ & 0.008 \\
\hline FDP & $1.20(0.80-1.60)$ & $1.20(0.90-1.60)$ & $1.20(0.80-1.60)$ & 0.607 \\
\hline DD & $0.27(0.17-0.43)$ & $0.29(0.17-0.43)$ & $0.26(0.17-0.43)$ & 0.742 \\
\hline \multicolumn{5}{|c|}{$\begin{array}{l}\text { Abbreviations: IQR, interquartile range; pCR, pathological complete response; PT, prothrombin time; } \\
\text { PTR, prothrombin time ratio; INR, international normalized ratio; APTT, activated partial } \\
\text { thromboplastin time; PTA, prothrombin activity; TT, thrombin time; Fib, fibrinogen; FDP, fibrinogen } \\
\text { degradation product; DD, D-dimer. }\end{array}$} \\
\hline
\end{tabular}

\section{Relationship between Fib status and clinicopathological characteristics}

ROC curve analysis and the Youden index were used to calculate the optimal cut-off value for Fib. Our results indicated that the optimal cut-off value for Fib was $3.145 \mathrm{~g} / \mathrm{L}(\mathrm{P}=0.008)$ (Figure 2B). Then, based on their low or high Fib status (Fib $<3.145 \mathrm{~g} / \mathrm{L}$ and $\geq 3.145 \mathrm{~g} / \mathrm{L}$ ), the patients were divided into groups as shown in Table 3. The relationships between Fib status and clinicopathological characteristics in our study were assessed by the chi-square test. Our results indicated that the low Fib status was significantly associated with premenopausal or perimenopausal status $(P<0.001), \leq 5 \mathrm{~cm}$ tumour size $(P=0.001)$, positive HR status $(P=0.003)$ and $>14 \% \mathrm{Ki} 67$ index $(P=0.028)$. However, we failed to detect relationships between Fib status and other clinical characteristics, including age $(P=0.299)$, clinical lymph node involvement status $(P=0.699)$, histological subtype $(P=0.503)$, and HER2 status $(P=$ 0.210). 
Table 3

Correlations of pre-treatment plasma fibrinogen status and clinicopathological features in breast cancer.

Fib status

Characteristics

Age (y)

$\leq 50$

$>50$

Menopausal status

Pre/peri

Post

Tumour size (cm)

$\leq 5$

$>5$

Lymph node involvement

No

Yes

Histologic subtype

ICD

No ICD

HR status*

Negative

Positive

HER2 status*

Negative

Positive

Ki67 index (\%)

$\leq 14$

$>14$

*63 cases with unknown HER2 status

Abbreviations: Fib, fibrinogen; ICD, invasive ductal carcinoma; HR, hormone receptor; HER2, human epidermal growth factor receptor 2 .
Low $(n=871)$

$443(57.5 \%)$

$328(42.5 \%)$

$42(53.8 \%)$

$122(46.2 \%)$

$<0.001$

$582(75.5 \%)$

$167(63.3 \%)$

$189(24.5 \%)$

$97(36.7 \%)$

0.001

$\begin{array}{ll}632(82.0 \%) & 192(72.7 \%) \\ 139(18.0 \%) & 72(27.3 \%)\end{array}$

0.699

\begin{tabular}{ll}
$276(35.8 \%)$ & $98(37.1 \%)$ \\
\hline $495(64.2 \%)$ & $166(62.9 \%)$
\end{tabular}

0.503

744 (96.5\%)

257 (97.3\%)

27 (3.5\%)

7 (2.7\%)

0.001

\begin{tabular}{ll}
$277(35.9 \%)$ & $124(47.0 \%)$ \\
\hline $494(64.1 \%)$ & $140(53.0 \%)$
\end{tabular}

385 (49.9\%)

147 (51.4\%)

334 (43.3\%)

106 (40.2\%)

0.210

0.028

$220(28.5 \%)$

57 (21.6\%)

551 (71.5\%)

207 (78.4\%)

P value

0.299

0.001




\section{Relationship between the clinicopathological features and PCR}

The chi-square test was used to assess the relationship between clinicopathological characteristics and pCR to NAC in breast cancer patients (Table 4). Clinicopathological factors were compared between the pCR group $(n=128)$ and the non-pCR group $(n=907)$. $p C R$ was correlated with $\leq 5 \mathrm{~cm}$ tumour size $(P=$ $0.009)$, no lymph node involvement $(P<0.001)$, negative HR status $(P<0.001),>14 \%$ Ki67 index $(P<$ $0.001)$ and low Fib status $(P=0.003)$. However, no significant differences in $\mathrm{pCR}$ were found in age $(P=$ $0.753)$, menopausal status $(P=0.257)$, histological subtype $(P=0.243)$, HER2 status $(P=0.280)$ and chemotherapy cycles $(P=0.870)$. 
Table 4

The relationship between the clinicopathological features and pathological complete response after neoadjuvant chemotherapy in breast cancer.

\begin{tabular}{|c|c|c|c|}
\hline & Responder & & \\
\hline Characteristics & Non-pCR $(n=907)$ & pCR $(n=128)$ & $P$ value \\
\hline Age (y) & & & 0.753 \\
\hline$\leq 50$ & $551(56.3 \%)$ & $74(57.8 \%)$ & \\
\hline$>50$ & $396(43.7 \%)$ & $54(42.2 \%)$ & \\
\hline Menopausal status & & & 0.257 \\
\hline Pre/peri & $651(71.8 \%)$ & $98(76.6 \%)$ & \\
\hline Post & $256(28.2 \%)$ & $30(23.4 \%)$ & \\
\hline Tumour size (cm) & & & 0.009 \\
\hline$\leq 5$ & $711(78.4 \%)$ & $113(88.3 \%)$ & \\
\hline$>5$ & $196(21.6 \%)$ & $15(11.7 \%)$ & \\
\hline Lymph node involvement & & & $<0.001$ \\
\hline No & $298(32.9 \%)$ & $76(59.4 \%)$ & \\
\hline Yes & $609(67.1 \%)$ & $52(40.6 \%)$ & \\
\hline Histologic subtype & & & 0.243 \\
\hline ICD & $875(96.5)$ & $126(98.4 \%)$ & \\
\hline No ICD & $32(3.5 \%)$ & $2(1.6 \%)$ & \\
\hline HR status & & & $<0.001$ \\
\hline Negative & $326(35.9 \%)$ & $75(58.6 \%)$ & \\
\hline Positive & $581(64.1 \%)$ & $53(41.4 \%)$ & \\
\hline HER2 status* & & & 0.280 \\
\hline Positive & $475(55.4 \%)$ & $57(50.0 \%)$ & \\
\hline Negative & $383(44.6 \%)$ & $57(50.0 \%)$ & \\
\hline Ki67 index (\%) & & & $<0.001$ \\
\hline$\leq 14$ & $264(29.1 \%)$ & $13(10.2 \%)$ & \\
\hline$>14$ & $643(70.9 \%)$ & $115(89.8 \%)$ & \\
\hline Chemotherapy cycles & & & 0.870 \\
\hline
\end{tabular}




\begin{tabular}{|c|c|c|c|}
\hline$<4$ & $16(1.8 \%)$ & $2(1.6 \%)$ & \\
\hline$\geq 4$ & $891(98.2 \%)$ & $126(98.4 \%)$ & \\
\hline Fib status & & & 0.003 \\
\hline Low & $662(73.0 \%)$ & $109(85.2 \%)$ & \\
\hline High & $245(27.0 \%)$ & $19(14.8 \%)$ & \\
\hline \multicolumn{4}{|c|}{ *63 cases with unknown HER2 status } \\
\hline \multicolumn{4}{|c|}{$\begin{array}{l}\text { Abbreviations: pCR, pathological complete response; ICD, invasive ductal carcinoma; HR, hormone } \\
\text { receptor; HER2, human epidermal growth factor receptor 2; Fib fibrinogen. }\end{array}$} \\
\hline
\end{tabular}

\section{Ability of Fib to predict pCR to NAC}

According to the Mann-Whitney $\mathrm{U}$ test and the chi-square test, the results indicated that PT, PTR, INR, APTT, PTA, TT, FDP, DD, age, menopausal status, histological subtype, HER2 status, and chemotherapy cycles were not significantly associated with pCR to NAC treatment (all P®0.05, Table 2 and Table 4). Considering clinical practice and statistical power, TT, Fib status, tumour size, lymph node involvement, HR status, HER2 status, Ki67 index, and chemotherapy cycles were included in the multivariate logistic regression analysis. The results indicated that Fib status $(P=0.002)$, tumour size $(P=0.001)$, lymph node involvement $(P<0.001)$, HR status $(P=0.001)$, and Ki67 index $(P<0.001)$ were independently correlated with the $\mathrm{pCR}$ to NAC. After adjusting for other factors in the logistic regression model, Fib was an independent predictive factor for PCR to NAC, and low Fib status (Fib $<3.145 \mathrm{~g} / \mathrm{L}$ ) was strongly associated with a better $\mathrm{pCR}$ rate $(\mathrm{OR}=2.365,95 \% \mathrm{Cl}=1.354-4.133, \mathrm{P}=0.002)($ Table 5$)$. 
Table 5

Logistic regression analysis of clinicopathological factors and pathological complete response after neoadjuvant chemotherapy in breast cancer.

\begin{tabular}{|c|c|c|c|}
\hline \multirow[t]{2}{*}{ Factor } & \multicolumn{3}{|c|}{ Multivariate analysis } \\
\hline & OR & $\mathrm{Cl} 95 \%$ & $P$ valve \\
\hline TT (continuous) & & & 0.988 \\
\hline Fib status (low vs high) & 2.365 & $1.354-4.133$ & 0.002 \\
\hline Tumour size ( $>5 \mathrm{~cm}$ vs $\leq 5 \mathrm{~cm}$ ) & 0.303 & $0.147-0.622$ & 0.001 \\
\hline Lymph node involvement (yes vs no) & 0.348 & $0.230-0.528$ & $<0.001$ \\
\hline HR status (positive vs negative) & 0.483 & $0.318-0.735$ & 0.001 \\
\hline HER2 status (positive vs negative) & & & 0.692 \\
\hline Ki67 index (> 14\% vs $\leq 14 \%$ ) & 3.274 & $1.694-6.329$ & $<0.001$ \\
\hline Chemotherapy cycles ( $\geq 4$ vs $<4$ ) & & & 0.915 \\
\hline
\end{tabular}

\section{Discussion}

Nowadays, NAC has become an integral part of the systematic treatment of breast cancer [1]. A major advantage of this strategy is the ability to observe the tumour response to chemotherapy regimens before surgery [2]. It is well known that the prognosis of patients with locally advanced breast cancer is closely related to whether $\mathrm{pCR}$ is achieved after NAC. Patients with $\mathrm{pCR}$ after NAC could have a better prognosis than those without $[3,4]$. In this study, we examined 1035 consecutive breast cancer patients who received NAC to provide evidence for the predictive value of Fib in pCR. The main finding of our analysis is that a lower pre-treatment Fib level is a significant independent predictor of $\mathrm{pCR}(\mathrm{OR}=2.365,95 \% \mathrm{Cl}=$ 1.354-4.133, $P=0.002)$ and was associated with premenopausal or perimenopausal status $(P<0.001), \leq$ $5 \mathrm{~cm}$ tumour size $(P=0.001)$, positive HR status $(P=0.003)$ and $>14 \% \mathrm{Ki} 67$ index $(P=0.028)$ in breast cancer patients.

Fib is a $340 \mathrm{kDa}$ glycoprotein that is mainly synthesized in the liver upon inflammatory stimulation by IL6 and IL-1 [9]. It is generally regarded as a key factor of coagulation and fibrinolytic activation. Recently, A lot of research shows that an elevated pre-treatment plasma Fib level is closely associated with poor prognosis in patients with various malignant tumours [18-21], including breast cancer[12-14]. In a large retrospective study of 2073 consecutive breast cancer patients, Wen et al. [12] reported that an elevated preoperative plasma Fib level was an independent prognostic factor for overall survival in breast cancer patients who underwent surgical treatment $(p=0.001)$. Additionally, a retrospective analysis including 520 
consecutive breast cancer patients found that an increased pre-treatment plasma Fib level was closely associated with shorter disease-specific survival $(p=0.042)$ [13].

Previous studies have demonstrated that Fib can also be endogenously synthesized by breast cancer cells themselves $[6,22]$. Several mechanisms can explain the contribution of Fib to tumour cell infiltration and expansion. First, due to the strong procoagulant effect of tumour cells, a large amount of Fib is aggregated around tumour cells and converted into fibrin, which is involved in metastasis and new vessel formation and promotes the formation of tumour stromal tissue [22, 23]. Second, Shu et al. demonstrated that high concentrations of Fib can induce epithelial-mesenchymal transition [24], which confers migration, invasion and metastasis capacities to tumour cells and renders tumour cells resistant to multiple drugs [25]. Moreover, Fib can promote tumour proliferation and stimulate angiogenesis through interactions with fibroblast growth factor 2 and vascular endothelial growth factor $[9,26]$. In addition, Fib can act as a bridge between the tumour and the host cell and support the adhesion of tumour cells to the vascular endothelium of target organs $[26,27]$.

Few studies have reported the relationship between the pre-treatment Fib level and pCR to NAC in breast cancer. In a study including 67 breast cancer patients receiving NAC, Mei et al revealed that patients who had decreased Fib levels after NAC had a better clinical response than patients who had stable or increased Fib levels[28]. In our centre, implantable venous access port systems or peripherally inserted central venous catheters are routinely provided to breast cancer patients before chemotherapy, which may promote the hypercoagulable state of the blood. The comparison of plasma Fib levels before and after chemotherapy may be unreliable. Therefore, we focused on the relationship between the pretreatment plasm Fib status and the PCR of NAC in breast cancer patients.

In our study, the median value of Fib was significantly increased in pCR patients compared with non-pCR patients (3.05 (2.63-3.48) g/L vs 2.90 (2.53-3.28) g/L, $P=0.008)$, while no significant differences in PT, PTR, INR, APTT, PTA, FDP, and DD were noted between the pCR group and the non-pCR group. Some studies suggest that DD may be related to the clinical stage and prognosis of breast cancer [29,30]. But we failed to observe the relationship between DD and pCR to NAC in breast patients. According to ROC curve analysis and the Youden index, the optimal cut-off value was $3.145 \mathrm{~g} / \mathrm{L}(\mathrm{P}=0.008)$. This value is discrepant with those previously reported[12-14] due to the differences in sample sources and predicted targets. A higher pCR rate was noted in the low Fib group (a value less than $3.145 \mathrm{~g} / \mathrm{L})(P=0.003)$. Adjusted for other clinicopathological factors in the logistic regression model, low Fib status was still strongly associated with a better $\mathrm{pCR}$ rate $(\mathrm{OR}=2.365,95 \% \mathrm{Cl}=1.354-4.133, \mathrm{P}=0.002)$. However, in 84 oesophageal cancer patients, higher pre-treatment plasma Fib levels were found to be significantly associated with a better histological response to neoadjuvant treatment [31]. This finding seems to contradict our results. This may be due to differences in sample size and tumour nature. Moreover, our results showed that lower plasma Fib levels were significantly associated with premenopausal or perimenopausal status $(P<0.001), \leq 5 \mathrm{~cm}$ tumour size $(P=0.001)$, positive HR status $(P=0.003)$ and $>$ $14 \% \mathrm{Ki} 67$ index $(P=0.028)$. These are similar to published studies about Fib and clinicopathological factors in breast cancer [12-14]. 
There were some limitations to this study. First, due to the retrospective nature of the current study, a selection bias is unavoidable. To limit interference factors, some exclusion criteria were set, but the relationship between these factors and breast cancer was not evaluated. Second, most patients received NAC for 4 cycles in our centre, so the pCR rates of this cohort were lower than those reported in other literature [32]. Third, long-term follow-up has not been completed of our cohort, so we cannot provide survival data for these patients to further study the relationship between the pre-treatment Fib level and the prognosis. However, this study may offer more evidence and recognition for the relationship between Fib and breast cancer and facilitate the administration of NAC therapy to achieve a better pCR rate. Further prospective trials are needed to confirm the predictive significance of Fib in breast cancer.

\section{Conclusions}

This study demonstrates that Fib was significantly associated with menopausal status, tumour size, ER status, PR status, and molecular subtype. More importantly, preoperative plasma low Fib status (Fib < $3.145 \mathrm{~g} / \mathrm{L}$ ) is an independent predictive factor for PCR to NAC in breast cancer patients. Breast cancer patients with low pre-treatment Fib levels should be recommended to receive NAC in order to achieve pCR and obtain clinical benefits.

\section{Abbreviations}

NAC, neoadjuvant chemotherapy; pCR, pathological complete response; Fib, Fibrinogen; TNM, tumournode-metastasis; ER oestrogen receptor; PR, progesterone receptor; HER2, human epidermal growth factor receptor 2; PT, prothrombin time; PTR, prothrombin time ratio; INR, international normalized ratio; APTT, activated partial thromboplastin time; PTA, prothrombin activity; TT, thrombin time; FDP, fibrinogen degradation product; $\mathrm{DD}$, D-dimer; HR, hormone receptor; IQR, interquartile range; $\mathrm{ROC}$, receiver operating characteristic; ORs, odds ratios; Cls, confidence intervals.

\section{Declarations}

\section{Ethics approval and consent to participate}

This research was conducted ethically in accordance with the World Medical Association Declaration of Helsinki and was approved by the Ethics Committee of our hospital (No. 2020-59), who deemed that written informed consent was not necessary due to the retrospective nature of the research.

\section{Consent to publication}

Not applicable.

\section{Availability of data and materials}


The data used and analyzed during the current study are available from the corresponding author on reasonable request.

\section{Competing interests}

The authors declare that they have no competing interests.

\section{Funding}

This work was supported by the National Natural Science Foundation of China [grant number 81272265]. The funding source had no involvement in study design, collection or analysis, and interpretation of data; nor in the writing of the report and in the decision to submit the article for publication.

\section{Author contributions}

Guarantor of integrity of the entire study: Shengchun Liu

Study concepts and design: Yihua Wang, Rui Chen, Zhenrong Tang, Shengchun Liu

Data acquisition: Yihua Wang, Yu Wang, Yang Peng

Quality control of data and algorithms: Yihua Wang, Yu Wang, Zhenrong Tang

Data analysis and interpretation: Yihua Wang, Yu Wang, Yang Peng, Zhenrong Tang

Statistical analysis: Yihua Wang, Yu Wang, Yang Peng

Manuscript preparation: Yihua Wang, Yu Wang

Manuscript editing: Yihua Wang, Yu Wang, Rui Chen, Zhenrong Tang, Shengchun Liu

Manuscript review: Yihua Wang, Yu Wang, Shengchun Liu

All authors have made substantial contributions to the conception and design of the study, or acquisition of data, or analysis and interpretation of data, and to the drafting the article or revising it critically for important intellectual content. All authors have read and approved the manuscript.

\section{References}

1. Bray F, Ferlay J, Soerjomataram I, Siegel RL, Torre LA, Jemal A. Global cancer statistics 2018 : GLOBOCAN estimates of incidence and mortality worldwide for 36 cancers in 185 countries. Cancer J Clin. 2018;68(6):394-424. 
2. Kaufmann M, von Minckwitz G, Mamounas EP, Cameron D, Carey LA, Cristofanilli M, Denkert C, Eiermann W, Gnant M, Harris JR, et al: Recommendations from an international consensus conference on the current status and future of neoadjuvant systemic therapy in primary breast cancer. Ann Surg Oncol 2012, 19(5):1508-1516.

3. von Minckwitz G, Untch M, Blohmer J-U, Costa SD, Eidtmann H, Fasching PA, Gerber B, Eiermann W, Hilfrich J, Huober J, et al. Definition and impact of pathologic complete response on prognosis after neoadjuvant chemotherapy in various intrinsic breast cancer subtypes. J Clin Oncol. 2012;30(15):1796-804.

4. Cortazar P, Zhang L, Untch M, Mehta K, Costantino JP, Wolmark N, Bonnefoi H, Cameron D, Gianni L, Valagussa $\mathrm{P}$, et al. Pathological complete response and long-term clinical benefit in breast cancer: the CTNeoBC pooled analysis. Lancet. 2014;384(9938):164-72.

5. Lyman GH, Khorana AA. Cancer, clots and consensus: new understanding of an old problem. J Clin Oncol. 2009;27(29):4821-6.

6. Falanga A, Marchetti M. Hemostatic biomarkers in cancer progression. Thromb Res. 2018;164(Suppl 1):54-61.

7. Batschauer APB, Figueiredo CP, Bueno EC, Ribeiro MA, Dusse LMS, Fernandes AP, Gomes KB, Carvalho MG. D-dimer as a possible prognostic marker of operable hormone receptor-negative breast cancer. Ann Oncol. 2010;21(6):1267-72.

8. Lal I, Dittus K, Holmes CE. Platelets, coagulation and fibrinolysis in breast cancer progression. Breast Cancer Res. 2013;15(4):207-7.

9. Tennent GA, Brennan SO, Stangou AJ, O'Grady J, Hawkins PN, Pepys MB. Human plasma fibrinogen is synthesized in the liver. Blood. 2007;109(5):1971-4.

10. Abdol Razak NB, Jones G, Bhandari M, Berndt MC, Metharom P. Cancer-Associated Thrombosis: An Overview of Mechanisms, Risk Factors, and Treatment. Cancers (Basel). 2018;10(10):380.

11. Falanga A, Marchetti M, Russo L. The mechanisms of cancer-associated thrombosis. Thromb Res. 2015;135:8-11.

12. Wen J, Yang Y, Ye F, Huang X, Li S, Wang Q, Xie X. The preoperative plasma fibrinogen level is an independent prognostic factor for overall survival of breast cancer patients who underwent surgical treatment. Breast. 2015;24(6):745-50.

13. Krenn-Pilko S, Langsenlehner U, Stojakovic T, Pichler M, Gerger A, Kapp KS, Langsenlehner T. An elevated preoperative plasma fibrinogen level is associated with poor disease-specific and overall survival in breast cancer patients. Breast. 2015;24(5):667-72.

14. Mei Y, Zhao S, Lu X, Liu H, Li X, Ma R. Clinical and Prognostic Significance of Preoperative Plasma Fibrinogen Levels in Patients with Operable Breast Cancer. PLoS One. 2016;11(1):e0146233-3.

15. Edge SB, Compton CC. The American Joint Committee on Cancer: the 7th edition of the AJCC cancer staging manual and the future of TNM. Ann Surg Oncol. 2010;17(6):1471-4.

16. Goldhirsch A, Wood WC, Coates AS, Gelber RD, Thürlimann B, Senn HJ. Panel m: Strategies for subtypes-dealing with the diversity of breast cancer: highlights of the St. Gallen International Expert 
Consensus on the Primary Therapy of Early Breast Cancer 2011. Ann Oncol. 2011;22(8):1736-47.

17. Mazouni C, Peintinger F, Wan-Kau S, Andre F, Gonzalez-Angulo AM, Symmans WF, Meric-Bernstam F, Valero V, Hortobagyi GN, Pusztai L. Residual ductal carcinoma in situ in patients with complete eradication of invasive breast cancer after neoadjuvant chemotherapy does not adversely affect patient outcome. J Clin Oncol. 2007;25(19):2650-5.

18. Man Y-N, Wang Y-N, Hao J, Liu X, Liu C, Zhu C, Wu X-Z. Pretreatment plasma D-dimer, fibrinogen, and platelet levels significantly impact prognosis in patients with epithelial ovarian cancer independently of venous thromboembolism. Int J Gynecol Cancer. 2015;25(1):24-32.

19. Lee S, Huh SJ, Oh SY, Koh MS, Kim S-H, Lee JH, Han JY, Choi HJ, Kim SJ, Kim H-J. Clinical significance of coagulation factors in operable colorectal cancer. Oncol Lett. 2017;13(6):4669-74.

20. Lin Y, Liu Z, Qiu Y, Zhang J, Wu H, Liang R, Chen G, Qin G, Li Y, Zou D. Clinical significance of plasma D-dimer and fibrinogen in digestive cancer: A systematic review and meta-analysis. Eur J Surg Oncol. 2018;44(10):1494-503.

21. Zhao L-Y, Zhao Y-L, Wang J-J, Zhao Q-D, Yi W-Q, Yuan Q, Chen X-Z, Li Y, Yang K, Chen X-L, et al: Is Preoperative Fibrinogen Associated with the Survival Prognosis of Gastric Cancer Patients? A Multicentered, Propensity Score-Matched Retrospective Study. World J Surg 2019:10.1007/s00268-0001905191-00269.

22. Simpson-Haidaris PJ, Rybarczyk B. Tumours and fibrinogen. The role of fibrinogen as an extracellular matrix protein. Ann N Y Acad Sci. 2001;936:406-25.

23. Kwaan HC, Lindholm PF. Fibrin and Fibrinolysis in Cancer. Semin Thromb Hemost. 2019;45(4):41322.

24. Shu Y-J, Weng H, Bao R-F, Wu X-S, Ding Q, Cao Y, Wang X-A, Zhang F, Xiang S-S, Li H-F, et al. Clinical and prognostic significance of preoperative plasma hyperfibrinogenemia in gallbladder cancer patients following surgical resection: a retrospective and in vitro study. BMC Cancer. 2014;14:566-6.

25. He L, Zhou X, Qu C, Hu L, Tang Y, Zhang Q, Liang M, Hong J. Musashi2 predicts poor prognosis and invasion in hepatocellular carcinoma by driving epithelial-mesenchymal transition. J Cell Mol Med. 2014;18(1):49-58.

26. Yano Hkitayama J, Hatano K, Tsuno N, Osada T, Watanabe T, Tsuruo T, Muto T, Nagawa H. Clustered cancer cells show a distinct adhesion behavior from single cell form under physiological shear conditions. J Exp Clin Cancer Res. 2001;20(3):407-12.

27. Steinbrecher KA, Horowitz NA, Blevins EA, Barney KA, Shaw MA, Harmel-Laws E, Finkelman FD, Flick MJ, Pinkerton MD, Talmage KE, et al. Colitis-associated cancer is dependent on the interplay between the hemostatic and inflammatory systems and supported by integrin alpha(M)beta(2) engagement of fibrinogen. Cancer Res. 2010;70(7):2634-43.

28. Mei Y, Liu H, Sun X, Li X, Zhao S, Ma R. Plasma fibrinogen level may be a possible marker for the clinical response and prognosis of patients with breast cancer receiving neoadjuvant chemotherapy. Tumour Biol. 2017;39(6):1010428317700002-2. 
29. Lu Y, Zhang L, Zhang Q, Zhang Y, Chen D, Lou J, Jiang J, Ren C: The association of D-dimer with clinicopathological features of breast cancer and its usefulness in differential diagnosis: A systematic review and meta-analysis. PloS one 2019, 14(9).

30. Batschauer A, Figueiredo C, Bueno E, Ribeiro M, Dusse L, Fernandes A, Gomes K, Carvalho M. D-dimer as a possible prognostic marker of operable hormone receptor-negative breast cancer. Ann Oncol. 2010;21(6):1267-72.

31. Ilhan-Mutlu A, Starlinger P, Perkmann T, Schoppmann SF, Preusser M, Birner P. Plasma fibrinogen and blood platelet counts are associated with response to neoadjuvant therapy in esophageal cancer. Biomark Med. 2015;9(4):327-35.

32. Spring L, Greenup R, Reynolds K, Smith BL, Moy B, Bardia A: Pathological complete response after neoadjuvant chemotherapy predicts improved survival in all major subtypes of breast cancer: systematic review and meta-analyses of over 18,000 patients. In.: AACR; 2016.

\section{Figures}

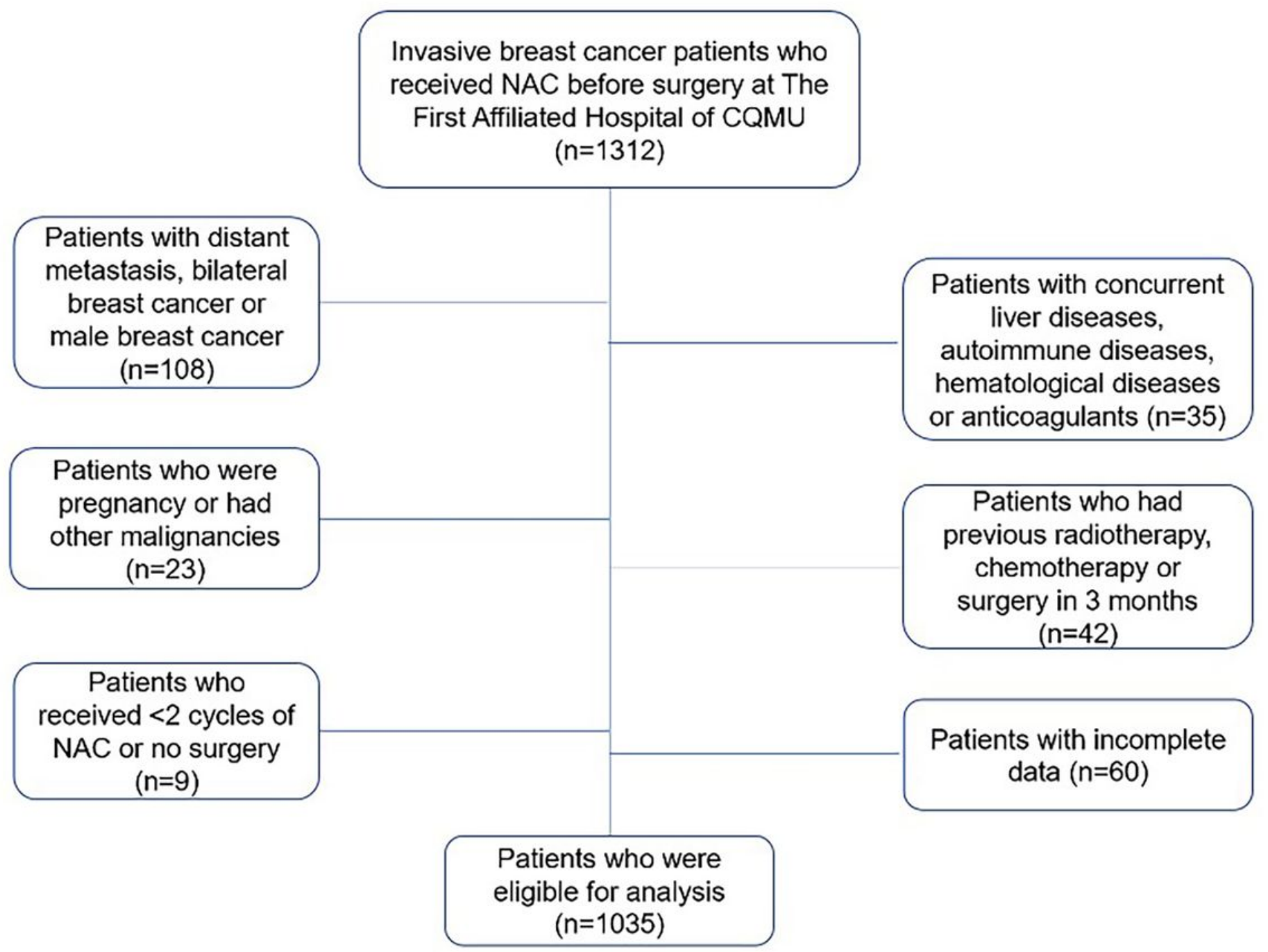

Figure 1 

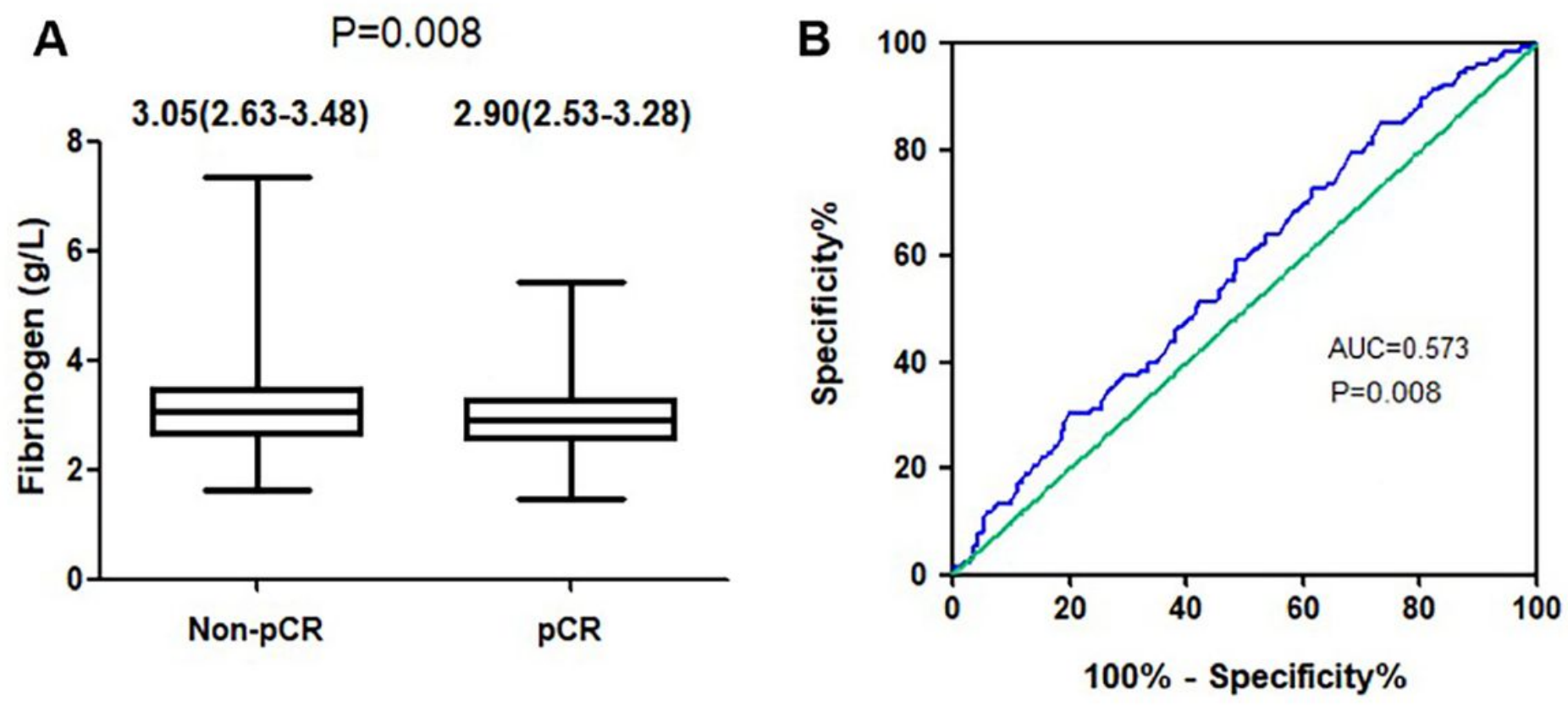

Figure 2

Critical analysis for Fib level for predicting pCR to NAC. Note: (A) Plasma Fib levels, expressed as medians and interquartile ranges, were significantly increased in non-pCR patients compared with pCR patients. (B) ROC curve analysis was used to evaluate the optimal cut-off value of preoperative Fib levels for predicting the pCR in the cohort study $(n=1035)$. The optimal Fib level cut-off value was identified according to the Youden index at $3.145 \mathrm{~g} / \mathrm{L}$. 